\title{
Benign simulators of melanoma on dermoscopy - black colour does not always indicate melanoma
}

\author{
Grazyna Kaminska-Winciorek', Jerzy Wydmanski'1,2 \\ ${ }^{1}$ The Center For Cancer Prevention and Therapy, Katowice, Poland \\ ${ }^{2}$ Department of Conventional and Intraoperative Radiotherapy Maria Skłodowska-Curie Memorial Cancer Center and \\ Institute of Oncology Gliwice Branch, Poland
}

Kaminska-Winciorek G, Wydmanski J. Benign simulators of melanoma on dermoscopy - black colour does not always indicate melanoma J Pre-Clin Clin Res. 2013; 7(1): 6-12.

\section{Abstract}

Introduction: Simulators of malignant melanoma comprise a heterogeneous group of melanocytic and nonmelanocytic lesions of the skin. They are mainly characterized by the occurrence of black or blue-black colors - both in clinical diagnosis as on dermoscopy. Therefore the proper recognition of this cases is extremely needed in daily general practice of many specialties' doctors.

Objective: The aim of the paper is the presentation of the most popular and possible simulators of malignant melanoma which have been accompanied by the occurrence of a black coloring on dermoscopy. This review presents a differential diagnosis of melanoma simulators and malignant melanoma based on Pubmed databes and own clinical experience.

State of knowledge: Paper reviews main potential benign melanoma-simulators such as melanocytic nevi (black nevus, blue nevus, and Spitz/Reed nevus), neoplasmatic proliferations (pigmented basal cell carcinoma - BCC and Kaposi sarcoma), vascular and hemorrhagic changes (labial venous lake, thrombosed hemangioma, pyogenic granuloma, subcorneal hematoma, and angiokeratoma), hyperkeratotic proliferations (seborrheic keratosis and melanoacanthoma) and miscellaneous (black comedo, cosmetic and posttraumatic tattoos). Authors described the main clinical and dermoscopic features of benign melanoma simulators.

Conclusions: The knowledge about melanoma simulators can reduce the number of surgical procedures and will help not only dermatologists, but also the professionals of other specialties, such as general practitioners, oncologists and also the paediatricians.

\section{- Key words}

dermoscopy, melanoma simulators, melanoma

\section{INTRODUCTION}

Dermoscopy is a diagnostic method based on global and local dermoscopic features. Differential diagnosis of many dermatologic entities has been proven by typical dermoscopic structures, their arrangement and colors.

Simulators of malignant melanoma comprise a heterogeneous group of melanocytic and nonmelanocytic lesions of the skin [1]. Melanoma simulators are mainly characterized by the occurrence of black or blue-black colors - both in clinical diagnosis as on dermoscopy. The occurrence of black coloring in dermoscopic images may be the result of a number of factors. Primarily, this black coloring may occur as a result of hemorrhagia with hemosiderin deposits inside the skin. The most popular reason for the appearance of this black coloring on dermoscopy is the accumulation of melanin grays in the dermis and epidermis - both of which are typical for pigmented lesions, including melanoma. This black color derivates also from the oxidized plug of keratin masses present in the sebaceous gland - whereas another reason may lie in the presence of pseudocysts in the seborrheic keratosis, or even papillomatous dermal nevi with

Address for correspondence: Grazyna Kaminska-Winciorek, Department of Conventional and Intraoperative Radiotherapy, Maria Skłodowska-Curie Memorial Cancer Center and Institute of Oncology Gliwice Branch, Wybrzeże Armii Krajowej 15, 44-101 Gliwice, Poland

e-mail: all4skin.eu@gmail.com

Received: 20 April 2013; accepted: 11 June 2013 multiple crypts. Black colors may result also from artificial tattoos - aesthetic tattoos or post-traumatic tattoos, e.g. those containing coal dust or gravel.

According to Scope et al. [2], a total of four non-melanocytic lesions also demonstrate homogenous blue pigmentation on dermoscopy, such as benign vascular lesions (usually hemangioma/angiokeratoma), Kaposi sarcoma, BCC, and radiation tattoos [2].

Objectives. The aim of the paper is the presentation of the most popular and possible dermatologic entities accompanied by the occurrence of black color on dermoscopy; however, they are not melanoma but only benign simulators of melanoma. The authors present a differential diagnosis of melanoma simulators and malignant melanoma based on own clinical experience and Pubmed databes. The knowledge of basic dermoscopic patterns of melanoma simulators, will help not only dermatologists, but also the professionals of other specialties, such as general practitioners, surgeons, oncologists and also the paediatricians.

\section{STATE OF KNOWLEDGE}

The melanoma mimickers are relatively common among multiple benign skin disorders from subcorneal hematoma to posttraumatic tattoo. The cases of melanoma simulators presented herein suggests a disturbingly melanoma. This is 
a clear example how important it is to take into account the entirety of the patient's medical history, performing clinical examination and dermoscopy to differentiate potential melanoma within its benign mimickers.

\section{MATERIALS AND METHOD}

We present the most popular and possible simulators of malignant melanoma which have been accompanied by the occurrence of a black coloring on dermoscopy. This review presents a differential diagnosis of melanoma simulators and malignant melanoma based on Pubmed databes and own clinical experience.

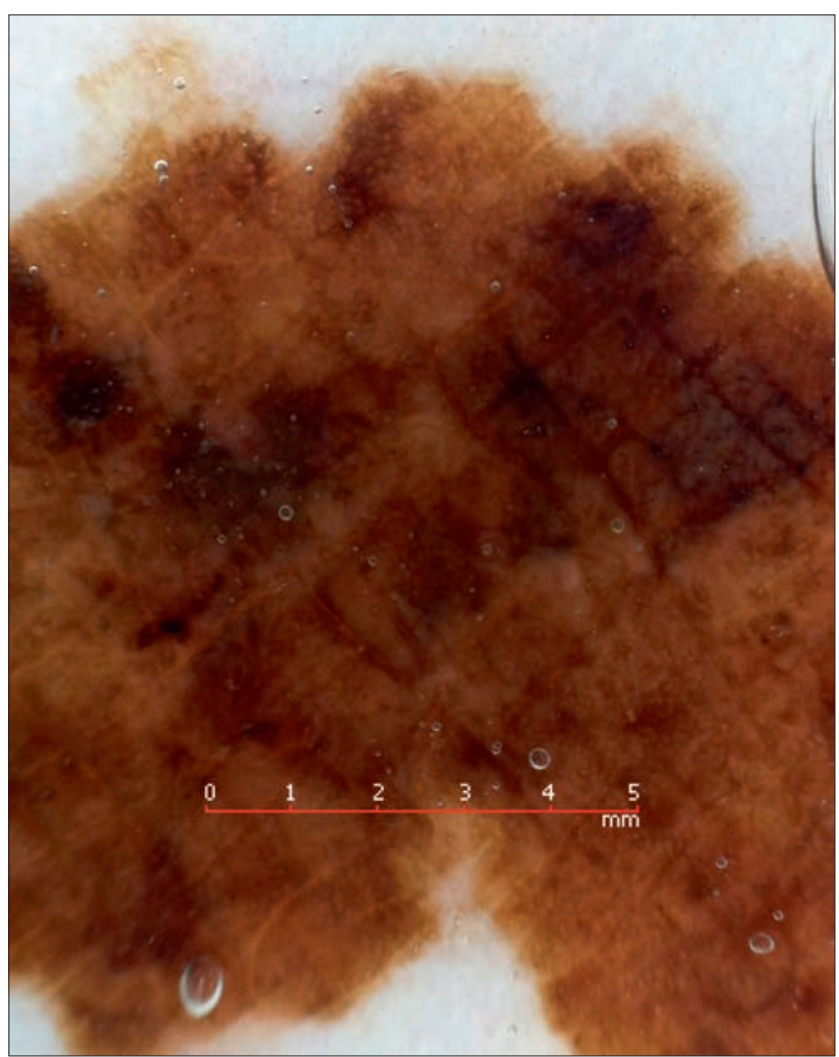

Figure 1. Lentigo melanoma on the left thigh; dermoscopy indicates the presence of atypical pigmented network, structureless homogenous blackish-brownish areas and atypical radial streaming

\section{RESULTS}

Primary melanoma. Although melanoma is a relatively common tumor, benign simulators of melanoma are extremely frequent. Atypical pigment networks, irregular globules and dots, irregular streaks, irregular pigmentation, regression structures (as a depigmented scarring area with significant peppering), a blue-whitish veil and vascular patterns - these are the most specific criteria for melanoma according to Neila and Soyer (Fig. 1, Fig. 2) [3]. Many histopathologic structures have been observed in melanoma, such as the presence of atypical melanocytes, nests of heavily pigmented melanocytes in the upper dermis which form under the acanthotic epidermis in a blue-white veil with accompanying peppering - pointing to zones with small multiple bluegray dots which in turn correspond to a variable number of melanophages in the papillary dermis have an influence on the image to be manifested by black or grey colors in the melanoma itself [3]. Black tinting has been observed in onethird of all slow-growing melanoma [4].

Dermoscopy is a very helpful diagnostic method, especially in the case of superficially spreading melanoma. However, it is less helpful in the case of black nodular melanoma [5]. Thus, a new dermoscopic criterion known as the blue-black rule has been proposed by Argenziano et al. [5] in 2011. The presence of blue and black colors on the dermoscopy of nodular lesions has a $78 \%$ sensitivity rate for melanoma - compared to a sensitivity of $43 \%$ achieved by standard melanoma criteria [5]. Figure 2A and Figure 2B present a nodular melanoma arising in the congenital nevus.

Metastatic melanoma. The metastatic melanoma is characterized by the occurrence of blue metastases, which sometimes were indistinguishable from the characteristic pattern of blue nevi (Fig. 3) [6]. In some rare cases of metastatic melanoma, only vascular structures in amelanocytic metastases can define its metastatic character [7].

Benign melanoma-simulators: Hemangioma. At present, dermoscopy allows for the differentiating of the infantile and cherry hemangiomas [8]. Oiso and Kamada [8] subclassifying proliferative infantile hemangiomas into superficial and thicker hemangioma, with a deeper component based on their dermoscopic images. Superficial hemangiomas have shown a polymorphous vascular structure without obvious red linear

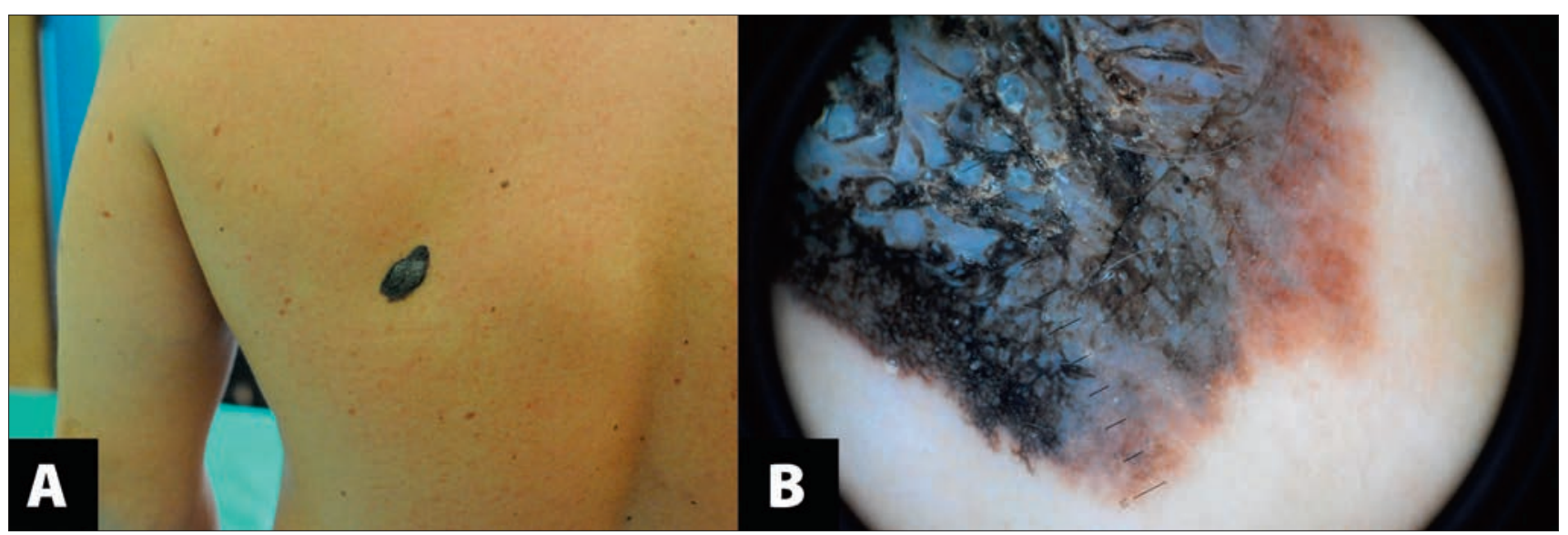

Figure 2. Melanoma arising from congenital nevus: A. macroscopic picture of the lesion. B. on dermoscopy atypical pigment networks, irregular pigmentation, regression structures (as a depigmented scarring with a blue-whitish veil) is seen 


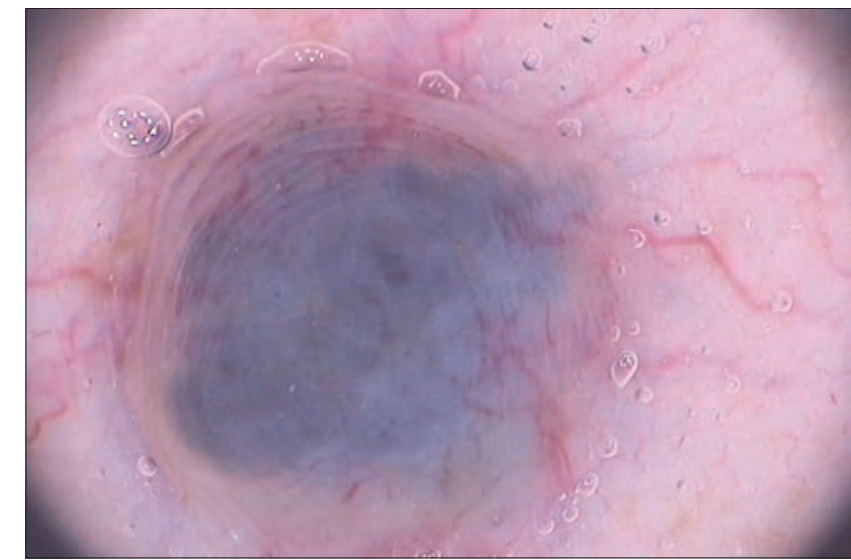

Figure 3. Metastatic melanoma: dermoscopy shows typical, homogenous bluishblackish pattern with circumflexing prominent vessels (courtesy of $\mathrm{Dr}$ Jacek Calik)

vessels and dilated red vessels [8]. The latter indicated the presence of polymorphous vascular structures with both linear red and dilated red vessels [8]. Typical dermoscopic images for cherry angioma are lacunar, homogenous, usually being violet or reddish in pattern [9]. In some cases of thrombotic hemangiomas, dermoscopic imaging suggests the presence of melanoma (Fig. 4). The thrombus inside the hemangioma is characterized by the presence of a jet-black color, occupying a large area [10]. In doubtful cases, the observer should find other specific features characteristic of hemangioma, such as red lagoons $[11,12]$ - in these cases, confocal microscopy can be performed [10].

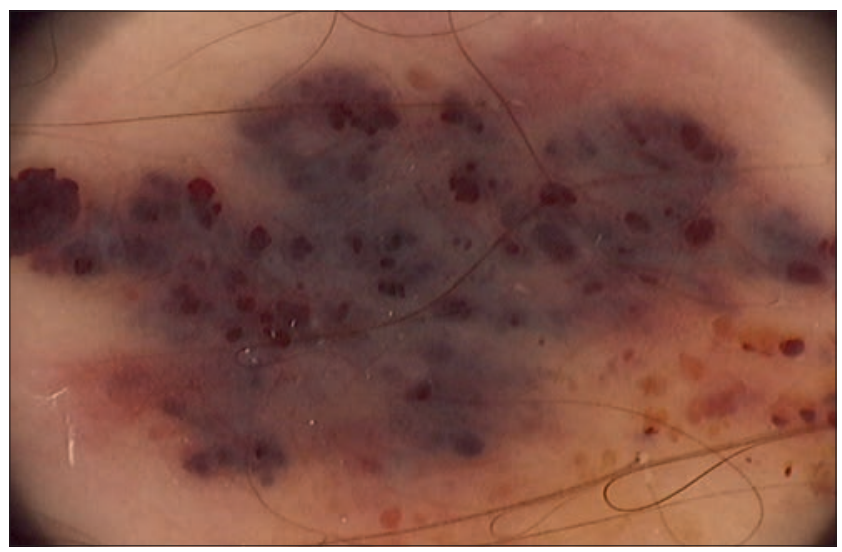

Figure 4. Thrombotic hemangioma: A typical dermoscopic image for cherry angioma is lacunar, homogenous pattern with the thrombus inside the hemangioma which is characterized by the presence of a jet-black color, occupying a large area of the lesion

Labial venous lake. In elderly hemangiomas on the lip on dermoscopy are revealed as bluish-reddish homogenous patterns [9]. They are sometimes thromboses, but after pressure sometimes they are partially vanished (Fig. 5).

Angiokeratoma. Angiokeratoma is characterized by dark violet to deep black homogenous colour and very often mimic melanoma (Fig. 6). According to Zaballos et al. [13] six dermoscopic structures were evident in at least $50 \%$ of the solitary angiokeratomas: dark lacunae (94\%), whitish veil (91\%), erythema (69\%), peripheral erythema (53\%), red lacunae (53\%), and hemorrhagic crusts (53\%). Dark lacunae exhibited a sensitivity of $93.8 \%$ and a specificity of $99.1 \%$, and dark lacunae and whitish veil, exhibited a sensitivity of

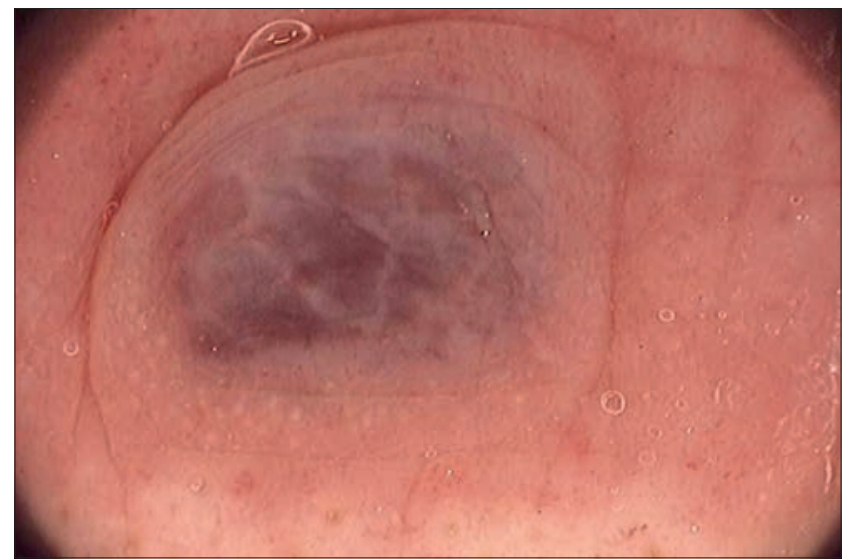

Figure 5. Labial venous lake: dermoscopy reveals the typical reddish-bluish homogenous pattern, without surrounding vessels

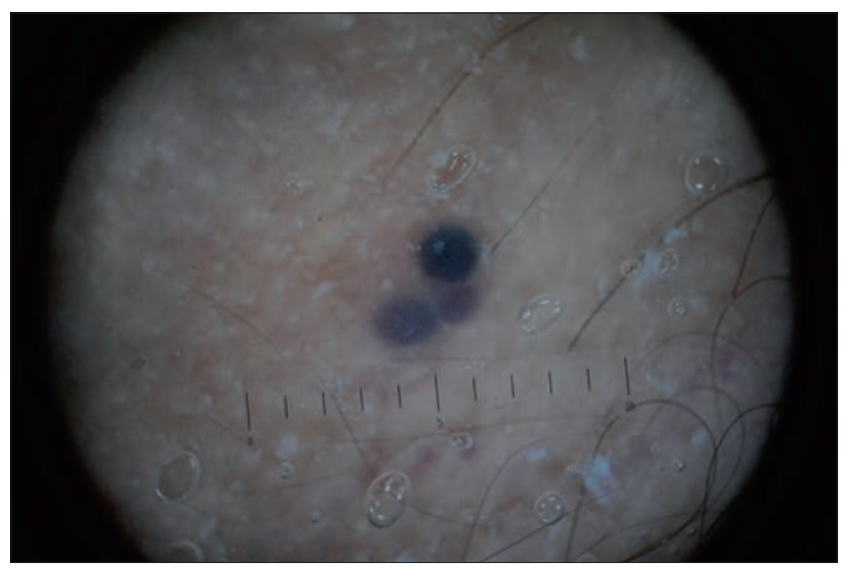

Figure 6. Angiokeratoma: on dermoscopy dark blackish lacunae with whitish areas are evident

$84.4 \%$ and a specificity of $99.1 \%$ not being found in malignant melanomas or pigmented basal cell carcinomas [13].

Pyogenic granuloma. Pyogenic granuloma is a common, benign, acquired, vascular growth of the skin and mucous membranes which is a simulator of amelanotic melanoma and other tumors. It is characterized by occurrence of four typical dermoscopic structures such as reddish homogeneous area, white collarette, white rail lines and vascular structures [14]. The absence of specific dermoscopic criteria for other skin tumors and a reddish homogeneous area surrounded by a white collarette are the most frequent dermoscopic pattern in pyogenic granulomas (85\%) [15]. However, occasionally this tumor can be difficult to differentiate clinically and dermoscopically from melanoma. Zaballos et al. [16] reported a case of pyogenic granuloma which strongly simulated melanoma because of its dermoscopic structures such as a black blotch, blue-white veil, polymorphous atypical vessels, milky-red areas, and hemorrhagic crusts [16].

Kaposi sarcoma. Kaposi sarcoma is characterized by occurrence of structureless bluish-reddish areas with typical rainbow sign and scaly surface which could be visualized on dermoscopy [11, 17] (Fig. 7).

Subcorneal hematoma. The most popular cause of black color developing in the skin is subcorneal hematoma. It 


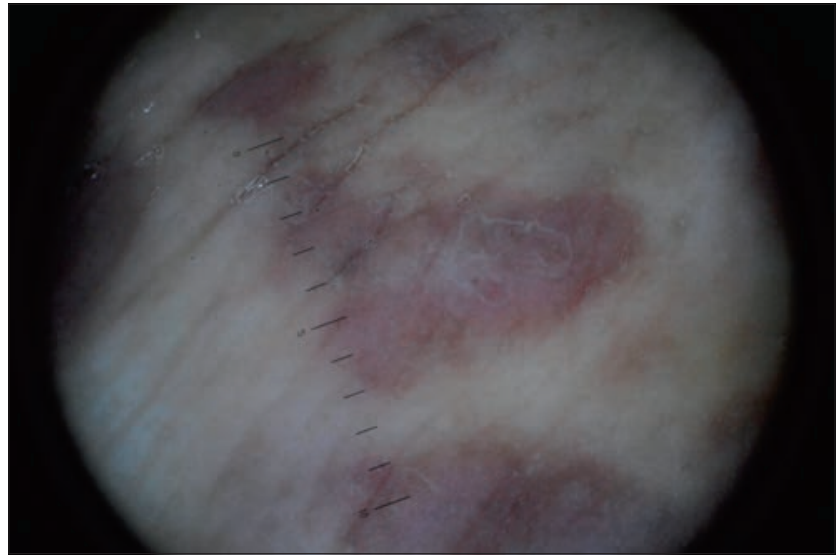

Figure 7. Kaposi sarcoma: dermoscopy is characterized by occurrence of structure less bluish-reddish areas, herein without typical rainbow sign and scaly surface

clinically characterizes by rapid manifestation, especially after trauma during sport activities. In many cases of subcorneal hematoma patient does not report the history and evaluation of the skin lesion. Sudden occurrence, especially as small black masses with homogenous black pattern on dermoscopy may threaten the patient. A dermoscopic examination usually reveals reddish-black homogeneous areas (Fig. 8), often accompanied by satellite globules [18]. In doubtful cases, a scraping test is pivotal for the confirmation of hematoma. The test is easy to perform and involves the gentle scraping off of the stratum corneum of the epidermis with a sterile scalpel or needle, which results in a complete or partial removal of the pigmentation in the parallel ridge pattern - a feature characteristic for hemorrhagic lesions acrally located, which is not observed in pigmented ones [18].

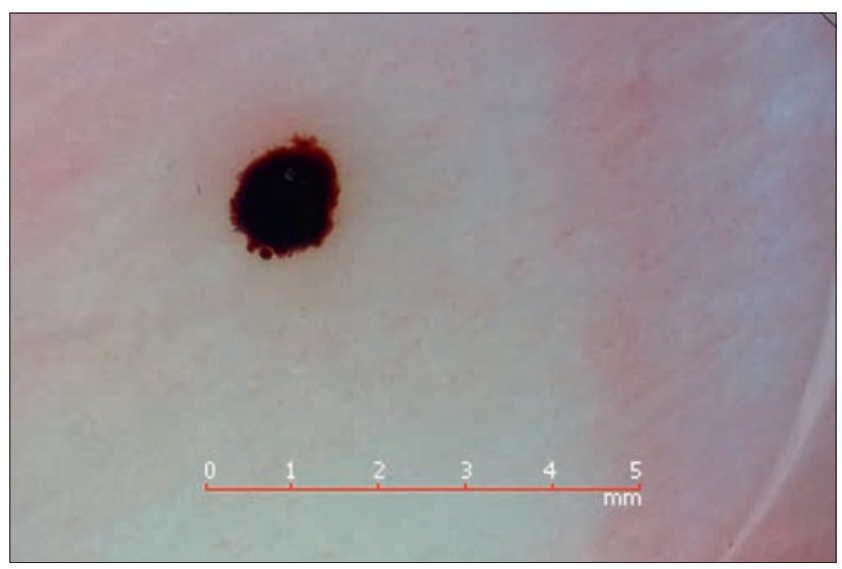

Figure 8. Subcorneal hematoma: a dermoscopic examination reveals reddishblack homogeneous areas accompanied by small satellite globules in the lower part of lesion

Hemorrhagic papilloma. In some cases after trauma some benign skin changes e.g. papilloma can transform its dermoscopic picture from brownish homogenous to black homogenous pattern. In such cases when occurring posttraumatic hemorrhages inside the skin lesion only history and histopathological examination help to distinguish benign lesion from melanoma.

Hemosiderotic dermatofibroma. Hemosiderotic dermatofibroma is a variant composed of numerous small vessels, extravasated erythrocytes and intra- and extracellular hemosiderin deposits [19]. On dermoscopy of hemosiderotic dermatofibroma a multicomponent pattern with a central bluish or reddish homogeneous area in combination with white structures and a peripheral delicate pigment network along with vascular structures is observed [19]. Hemosiderotic variant of dermatofibroma is extremely rare. In Kaarslan's study [20] homogeneous, blue-gray pigmentation (mainly in at the center focally) was observed only in 3 dermatofibromas (5.9\%). The most frequently described pitfalls are a central bluish or reddish homogeneous area with white structures and a peripheral light pigment network with vascular structures [21]. When on dermoscopy patognomic structures such as central white patch and delicate pigment network are absent the melanoma diagnosis could be made [22]. Because of presence of multicomponent pattern in some melanomas, indicated dermoscopic features of hemosiderotic dermatofibroma may not be specific to rule out the malignant neoplasm [19].

Melanocytic nevi. According to the newest methods of classification, melanocytic nevi are divided into 7 main groups: 1. globular/cobblestone pattern nevus; 2 . reticular nevus; 3 . starburst pattern nevus; 4 . homogenous blue nevus; 5. special- site located nevus; 6 . characteristic-features nevi; 7. unclassified melanocytic proliferations [23]. Dermoscopic diagnosis of nevi relies on the following four criteria (each of which is characterized by 4 variables): 1. color (black, brown, gray, or blue); 2. pattern (globular, reticular, starburst, and homogeneous blue pattern); 3. pigment distribution (multifocal, central, eccentric, and uniform); and 4. special sites (face, acral areas, nail, and mucosa). In addition, the following 6 factors related to the patient might influence the pattern of pigmentation of the individual nevi: age, skin type, history of melanoma, UV exposure, pregnancy, and growth dynamics [24].

The most common melanoma simulator are the blue nevus and the Spitz/Reed nevus [25].

Blue nevus. The blue nevus derives its name from its characteristic blue-gray color, resembling that of a copy pencil.

Patients often seem to believe that the occurrence of this change is related to trauma or ink pen staining $[26,27]$.The blue nevus frequently appears directly after birth or in early childhood.

The blue nevus appears as a blue-gray macule or nodule, usually taking the shape of a homogeneous structure. On dermoscopy, areas of homogeneous blue-gray pigmentation are dominant (Fig. 9), sometimes in a combination of colors: white, brown and gray.

According to the classification system of Ferrara et al. there are five main varieties of blue nevi: blue (the common blue nevus), white (the desmoplastic blue nevus), black (the Kamino nevus), brown (the combined blue nevus) and polychromic (the deep penetrating nevus) [28]. Up to $80 \%$ of blue nevi presented a homogeneous pattern consisting of one color (blue, black, or brown) or two colors (blue-brown, blue-gray, or blue-black) [5].

Literature has been known to describe a number of cases of blue nevi with a pattern different than that of the homogeneous, for example, a reminiscent reticular one [29]. At present, it is believed that ultrasonography plays an 


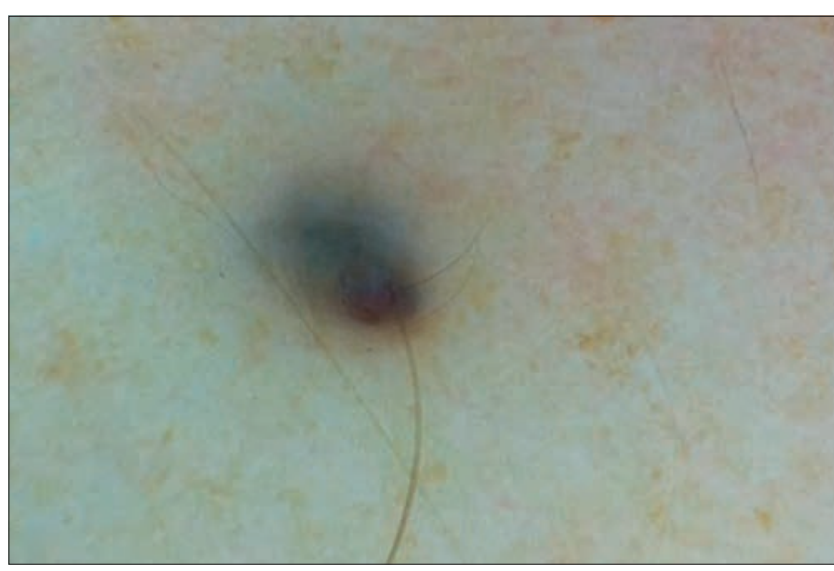

Figure 9. Blue nevus: on dermoscopy, areas of homogeneous blue-gray pigmentation are dominant

important role in the differential diagnosis of melanomas and blue nevi, as it possesses a high specificity (94\%) compared to clinical diagnosis (77\%) and dermoscopy (77\%) [30].

Spitz/ Reed nevus. The Spitz nevus (also known as benign juvenile melanoma) can often be clinically, dermoscopically and histologically confused with melanoma. This benign tumor is most commonly observed in childhood, with $70 \%$ of cases being diagnosed during the first 20 years of life. Both sexes are equally affected [31]. Among children, the Spitz nevus is usually located on the lower limbs, the trunk, the head and neck. It occurs in several clinical forms - first, as a bright pink nodule on the child's face (the classic Spitz nevus). It can metamorphose into a pink plaque, with central hyperpigmentation $[26,27]$. Sometimes, it appears as a bluegray spot (Reed nevus) - most often observed among young adults in the upper part of the chest and the lower extremities, which can often have a tendency for nodular growth, the creation of an uneven surface of the presence of various coloring. On dermoscopy, in most cases, Spitz nevi present a uniform distribution of pseudopods around the lesion, forming a "starburst pattern" (Fig. 10) [26, 27, 32].

Black nevus. Individuals with the IV skin type exhibit a predominant nevus-type, characterized by a dark brown color and central hyperpigmentation (the so-called black or hypermelanotic nevi) (Fig. 11) [24].

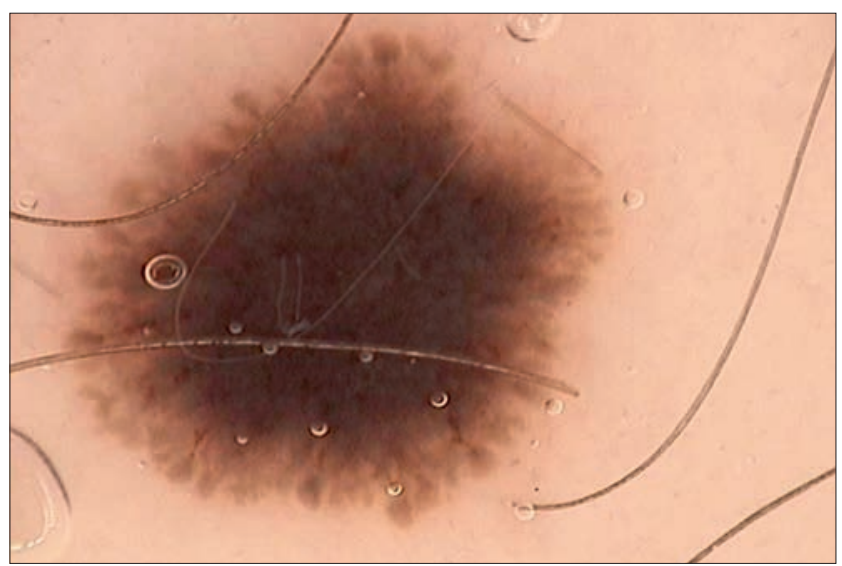

Figure 10. Spitz/Reed nevus: dermoscopy presents a uniform distribution of pseudopods around the lesion, forming a "starburst pattern"

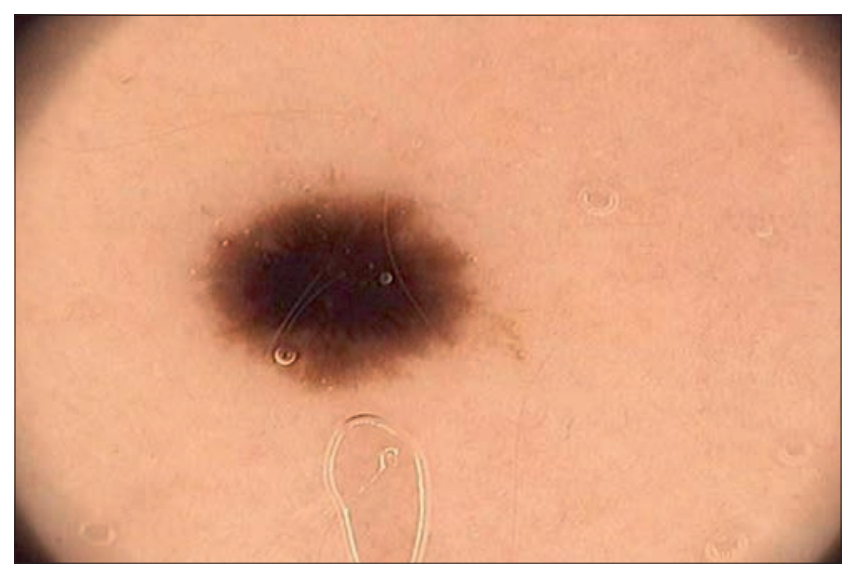

Figure 11. Black nevus: dermoscopic picture correlates with a predominant nevustype, characterized by a dark brown color and central hyperpigmentation

Targetoid hemosiderotic nevus. Among frequent clinical melanoma, simulators are injured melanocytic nevi [1] Some typical features of posttraumatic melanocytic nevi include a targetoid-halo on a long-lasting, acquired exophytic nevus with a hemorrhagic ring [26]. Usually, the targetoid nevus can pass into spontaneous regression within 4 weeks [33].

Dermal nevus. In some cases, particularly among the darker phototype skin individuals - black cobblestone or globules may suggest melanoma, especially when a hyperkeratosis and a scaling on the nevus surface has been observed (Fig. 12). In the case of verrucous nevi with crypt-like depressions filled with yellowish-blackish hyperkeratotic masses, a 5\% urea cream can be applied to the lesions for several days before dermoscopy, in order to dissolve the keratin masses [34].

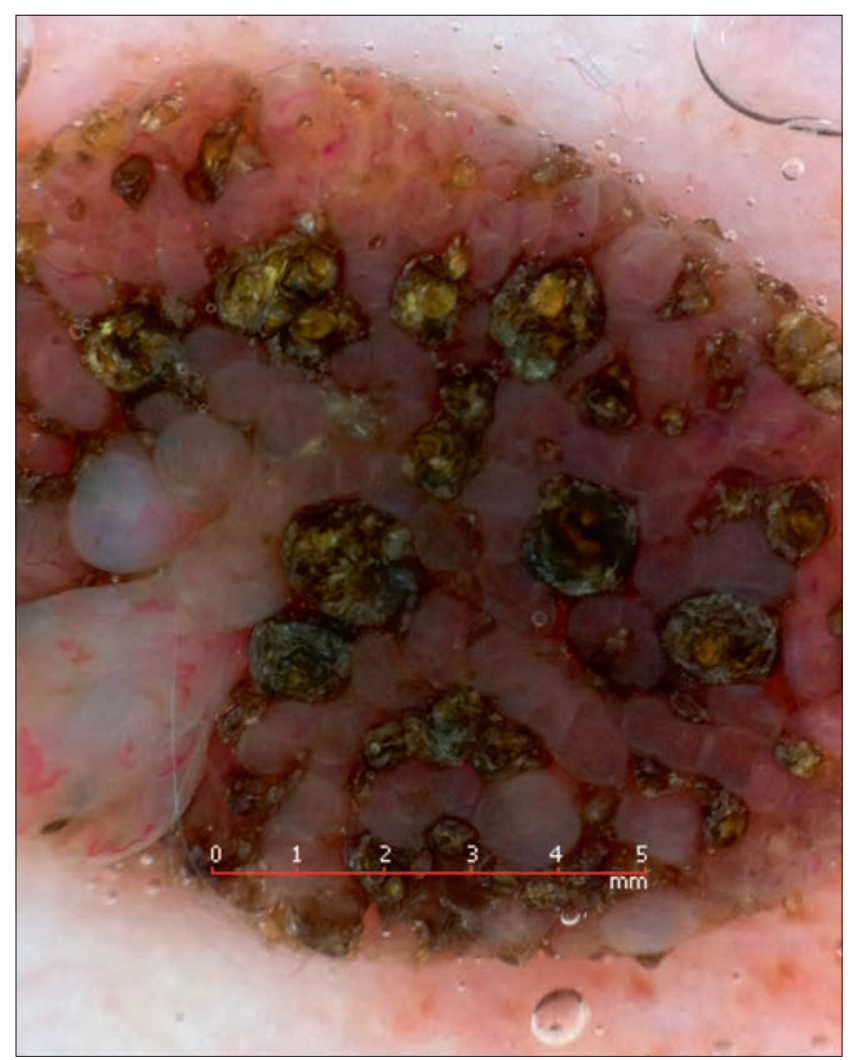

Figure 12. Dermal nevus: black homogenous pattern with crypts filled with blackish hyperkeratotic masses may suggest melanoma on dermoscopy 
Basal cell carcinoma (BCC). According to Menzies et al. [35] pigmented BCC to be diagnosed, the negative feature of pigment network is not permitted and the specimen must present one or more of the following six positive features: large, gray-blue ovoid nests, multiple gray-blue globules, maple leaf-like areas, spoke wheel areas, ulceration and arborizing "treelike" vessels [35]. The presence of bluishblackish structures such as ovoid nests, maple-leaf areas and grey-blue globules can increase the suspicion index of melanoma (Fig. 13). When melanin from the tumor nest, as well as the stroma, is remarkably increased, the nests form an unstructured pigmented area (a "blotch"), which can impair the diagnosis of BCC [36].

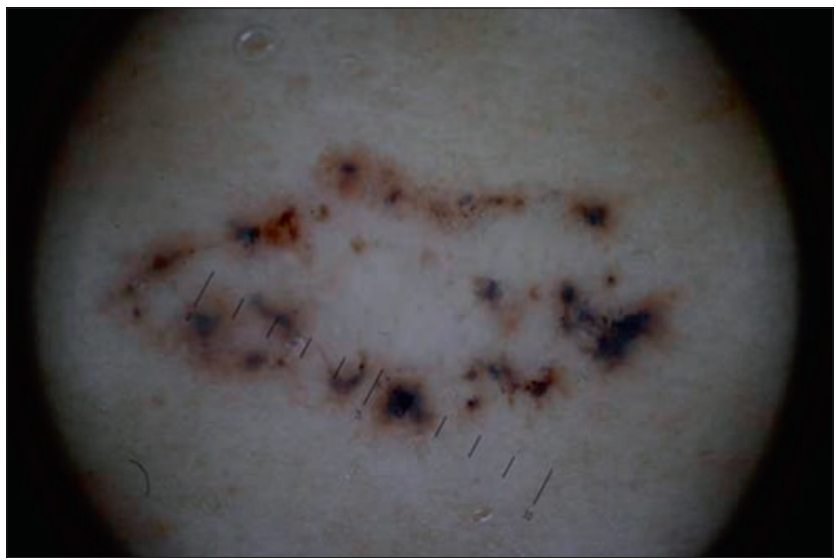

Figure 13. Pigmented BCC: dermoscopy reveals the presence of large, gray-blue ovoid nests, multiple gray-blue globules and maple leaf-like areas

Seborhheic keratosis. A typical dermoscopic picture of seborrheic keratosis shows the presence of comedolike openings and milia-like cysts, which easily allow the recognition of this entity [9]. In the pigmented variant of seborrheic keratosis, doctors can be slightly confused because of the melanoma simulator [37]. On dermoscopy, blackishbrownish homogenous structures are present (Fig. 14) [37]. When an atypical blackish, solitary lesion suggesting seborrheic keratosis is present, a total surgical excision can be performed - in order to avoid a melanoma misdiagnosis [33].

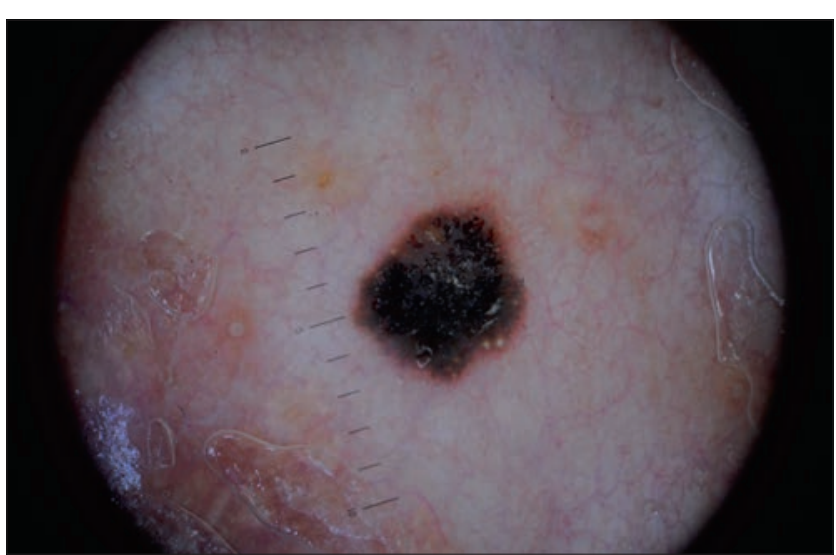

Figure 14. Seborrheic keratosis: on dermoscopy, blackish-brownish homogenous structures are present with several small milia-like cysts in the lower pole

Melanoacanthoma. Melanoacanthoma is a rare variant of the pigmented seborrheic keratosis (Fig. 15) [38]. In difficult diagnostic cases, on dermoscopy, regular pigment network

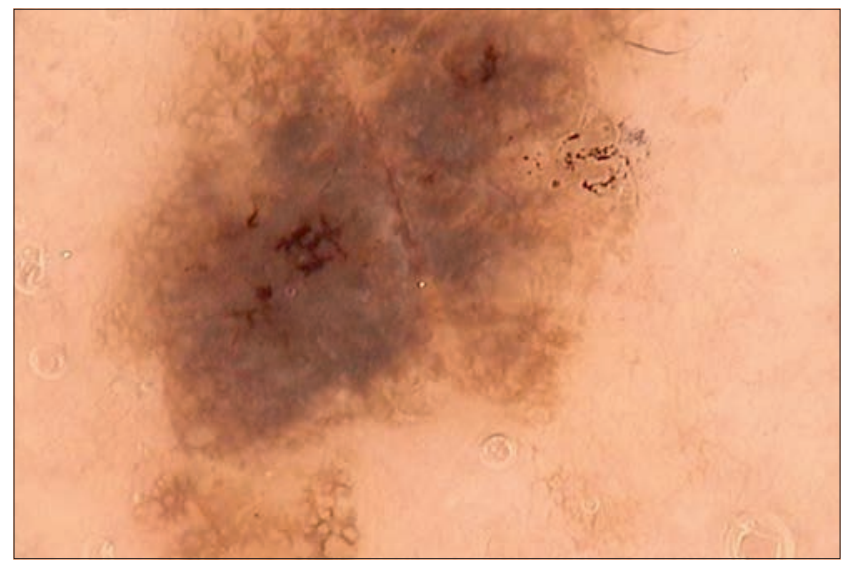

Figure 15. Melanoacanthoma is characterized by occurrence of blackish homogenous pattern, at 3 o'clock atypical remnants of pigment are seen

and black cribriform pattern of ridges have been observed [38]. On a rare occasion, the starburst pattern can be observed in the melanoacanthoma, mimicking the Spitz nevus and the melanoma [39].

Macrocomedones. Macrocomedone is a little cyst, accompanied by an enlarged ostium of the sebaceous gland, resulting especially from trauma. It is characterized by deposits of keratin masses inside the ostium, with a tendency for the oxidation of keratin plugs, forming brownish or black, homogenous, structureless areas. In some cases, it presents itself as a homogenous, structureless area, over which the whitish-blue hue is visible, corresponding to the blue pseudoveil sign [40]. After a slight pressure of the macrocomedone on the hyperkeratotic, these structureless masses are emptied and the 'blue pseudo-veil sign' subsequently vanishes. Bluish and whitish keratin masses are located under the skin, translucent, in the form of bluish pseudo-veil structures imitating melanoma [40].

Miscellaneous: Cosmetic and posttraumatic tattoos. In some cases, dermoscopy can be helpful, preceding the use of lasertherapy on posttraumatic or cosmetic tattoos [41]. In such cases, a bluish-blackish homogenous pattern has been observed on dermoscopy (Fig. 16) [2]. The deeper location of

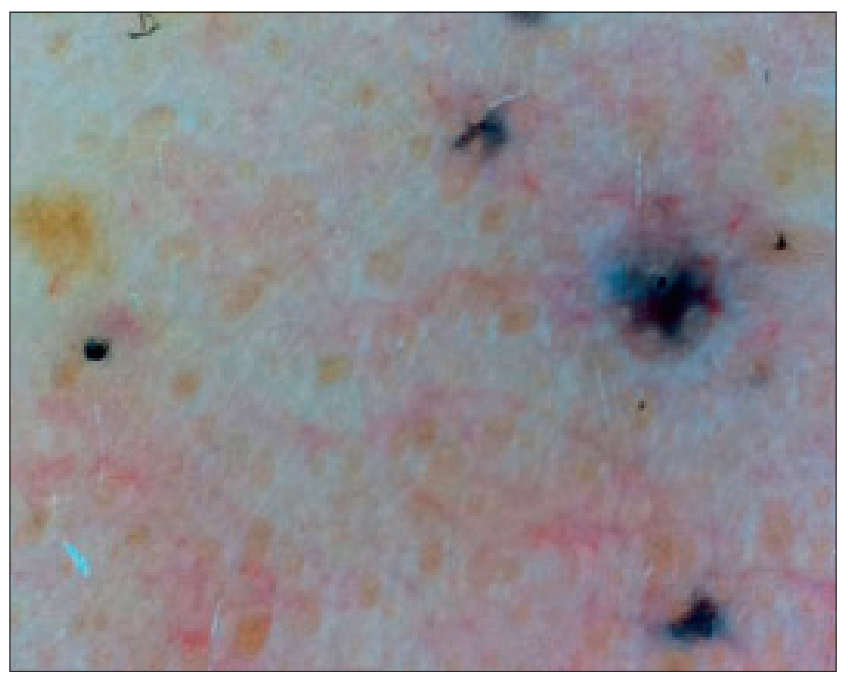

Figure 16. Posttraumatic tattoo: dermoscopy indicated many dispersed bluishblackish homogenous structures corresponding to gravel dust particles 
the ink in the dermis causes a bluish appearance, similarly to that of the dermal melanin, this once again being the effect of dermal scattering within the blue-shaded area [2].

\section{REFERENCES}

1. Tomasini C, Broganelli P, Pippione M. Targetoid hemosiderotic nevus. A trauma-induced simulator of malignant melanoma. Dermatology 2005; 210: 200-205.

2. Scope A, Benvenuto-Andrade C, Agero AL, Marghoob AA. Nonmelanocytic lesions defying the two-step dermoscopy algorithm. Dermatol Surg. 2006; 32: 1398-1406.

3. Neila J, Soyer HP. Key points in dermoscopy for diagnosis of melanomas, including difficult to diagnose melanomas, on the trunk and extremities. J Dermatol. 2011; 38: 3-9.

4. Terushkin V, Dusza SW, Scope A, Argenziano G, Bahadoran P, Cowell $\mathrm{L}$, et. al. Changes observed in slow-growing melanomas during longterm dermoscopic monitoring. Br J Dermatol. 2012; 166: 1213-1220.

5. Argenziano G, Longo C, Cameron A, Cavicchini S, Gourhant JY, Lallas A, et al. Blue-black rule: a simple dermoscopic clue to recognize pigmented nodular melanoma. Br J Dermatol. 2011; 165: 1251-1255.

6. Carlos-Ortega B, de Oca-Monroy FM, Isyta-Morales A. [Dermoscopy findings in malignant skin melanoma with metastases. A case report] Rev Med Inst Mex Seguro Soc. 2009; 47: 73-76.

7.Minagawa A, Koga H, Sakaizawa K, Sano K, Saida T. Dermoscopic and histopathological findings of polymorphous vessels in amelanotic cutaneous metastasis of pigmented cutaneous melanoma. Br J Dermatol. 2009; 160: 1134-1136.

8. Oiso N, Kawada A. The dermoscopic features in infantile hemangioma. Pediatr Dermatol. 2011; 28: 591-593.

9. Kamińska-Winciorek G. Digital dermatology.1 ${ }^{\text {st }}$ ed. Cornetis (Wroclaw), 2008.

10. Moscarella E, Zalaudek I, Buccini P, Cota C, Catricalà C, Argenziano G. Dermoscopy and confocal microscopy of thrombosed hemangiomas. Arch Dermatol. 2012; 148: 410.

11. Coates D, Bowling J. Dermoscopy is not always helpful in the diagnosis of vascular lesions. Australas J Dermatol. 2010; 51: 292-294.

12. Wang SQ, Katz B, Rabinovitz H, Kopf AW, Oliviero M. Lessons on dermoscopy \#7. The diagnosis was thrombosed hemangioma. Dermatol Surg. 2000; 26: 891-892.

13.Zaballos P, Daufí C, Puig S, Argenziano G, Moreno-Ramírez D, Cabo $\mathrm{H}$, et al. Dermoscopy of solitary angiokeratomas: a morphological study. Arch Dermatol. 2007; 143: 318-25.

14. Zaballos P, Carulla M, Ozdemir F, Zalaudek I, Bañuls J, Llambrich A, et al. Dermoscopy of pyogenic granuloma: a morphological study. Br J Dermatol. 2010; 163: 1229-1237.

15.Zaballos P, Llambrich A, Cuéllar F, Puig S, Malvehy J. Dermoscopic findings in pyogenic granuloma Br J Dermatol. 2006; 154: 1108-1111.

16. Zaballos P, Rodero J, Serrano P, Cuellar F, Guionnet N, Vives JM. Pyogenic granuloma clinically and dermoscopically mimicking pigmented melanoma Dermatol Online J. 2009; 15: 10.

17. Hu SC, Ke CL, Lee CH, Wu CS, Chen GS, Cheng ST. Dermoscopy of Kaposi's sarcoma: areas exhibiting the multicoloured 'rainbow pattern'. J Eur Acad Dermatol Venereol. 2009; 23: 1128-1132.

18. Zalaudek I, Argenziano G, Soyer HP, Saurat JH, Braun RP. Dermoscopy of subcorneal hematoma. Dermatol Surg. 2004; 30: 1229-1232.

19.Zaballos P, Llambrich A, Ara M, Olazarán Z, Malvehy J, Puig S. Dermoscopic findings of haemosiderotic and aneurysmal dermatofibroma: report of six patients. Br J Dermatol. 2006; 154: 244-250.
20. Kilinc Karaarslan I, Gencoglan G, Akalin T, Ozdemir F. Different dermoscopic faces of dermatofibromas. J Am Acad Dermatol. 2007; 57: 401-406.

21. Scalvenzi M, Balato A, De Natale F, Francia MG, Mignogna C, De Rosa G. Hemosiderotic dermatofibroma: report of one case. Dermatology 2007; 214: 82-4.

22. Blum A, Jaworski S, Metzler G, Bauer J. Lessons on dermoscopy: Dermoscopic pattern of hemosiderotic dermatofibroma. Dermatol Surg. 2004; 30: $1354-1355$.

23.Zalaudek I, Manzo M, Savarese I, Docimo G, Ferrara G, Argenziano G. The morphologic universe of melanocytic nevi. Semin Cutan Med Surg. 2009; 28: 149-156.

24.Zalaudek I, Docimo G, Argenziano G. Using dermoscopic criteria and patient-related factors for the management of pigmented melanocytic nevi. Arch Dermatol. 2009; 145: 816-826.

25. Kaminska-Winciorek G, Spiewak R. Basic dermoscopy of melanocytic lesions for beginners. Postepy Hig Med Dosw. (Online). 2011; 65: 501508 .

26. Kaminska-Winciorek G. Ultaviolet exposure and melanocytic nevi in children- prophylaxis and management. In: Otto-Buczkowska E. What's new in pediatrics? $2^{\text {nd }}$ ed. Wroclaw Cornetis, 2011.p. 491-501.

27. Kaminska-Winciorek G. Melanocytic nevi in children in its clinical and dermoscopic aspects. Dermatol Dziecięca. 2011; 1: 68-74.

28. Ferrara G, Soyer HP, Malvehy J, Piccolo D, Puig S, Sopena J, et al. The many faces of blue nevus: a clinicopathologic study. J Cutan Pathol. 2007, 34: 543-551.

29. Tsunemi Y, Saeki H, Tamaki K. Blue naevus with pigment network-like structure on dermoscopy. Acta Derm Venereol. 2008; 88: 412-413.

30. Samimi M, Perrinaud A, Naouri M, Maruani A, Perrodeau E, Vaillant L, et al. High-resolution ultrasonography assists the differential diagnosis of blue naevi and cutaneous metastases of melanoma. Br J Dermatol. 2010; 163: 550-556.

31. Requena C, Requena L, Kutzner H, Sánchez Yus E. Spitz nevus: a clinicopathological study of 349 cases. Am J Dermatopathol. 2009; 31: 107-116.

32. Ferrara G, Zalaudek I, Savarese I, Scalvenzi M, Argenziano G. Pediatric atypical spitzoid neoplasms: a review with emphasis on 'red' ('spitz') tumors and 'blue' ('blitz') tumors. Dermatology. 2010; 220: 306-10.

33. Patrizi A, Giacomini F, Savoia F, Misciali C, Neri I. Targetoid hemosiderotic naevus. J Eur Acad Dermatol Venereol. 2009; 23: 493-494.

34. Kaminska-Winciorek G, Spiewak R. Tips and tricks in the dermoscopy of pigmented lesions. BMC Dermatol. 2012; 12: 14.

35. Menzies SW. Dermoscopy of pigmented basal cell carcinoma. Clin Dermatol. 2002; 20: 268-269.

36. Takenouchi T. Key points in dermoscopic diagnosis of basal cell carcinoma and seborrheic keratosis in Japanese. J Dermatol. 2011; 38: 59-65.

37. de Giorgi V, Massi D, Salvini C, Mannone F, Carli P. Pigmented seborrheic keratoses of the vulva clinically mimicking a malignant melanoma: a clinical, dermoscopic-pathologic case study. Clin Exp Dermatol. 2005; 30: 17-19.

38. Shankar V, Nandi J, Ghosh K, Ghosh S. Giant melanoacanthoma mimicking malignant melanoma. Indian J Dermatol. 2011; 56: 79-81.

39. Rossiello L, Zalaudek I, Ferrara G, Docimo G, Giorgio CM, Argenziano G. Melanoacanthoma simulating pigmented Spitz nevus: an unusual dermoscopy pitfall. Dermatol Surg. 2006; 32: 735-737.

40. Kaminska-Winciorek G. Letter: "Blue Pseudo-Veil Sign” - a New Dermoscopic Term? Dermatol Surg. 2012; 38:1574-1575.

41. Kaminska-Winciorek G, Spiewak R. [Dermoscopy in aesthetic medicine]. Polish Journ Cosmetol. 2011; 14: 17-26. 